\title{
Synergistic Inhibition of Kinase Pathways Overcomes Resistance of Colorectal Cancer Spheroids to Cyclic Targeted Therapies
}

\author{
Pradip Shahi Thakuri, ${ }^{\dagger}$ Megha Gupta, ${ }^{\ddagger}$ Ramila Joshi, ${ }^{\dagger}$ (๑) Sunil Singh, ${ }^{\dagger}$ and Hossein Tavana* ${ }^{\dagger} \oplus$ \\ ${ }^{\dagger}$ Department of Biomedical Engineering, The University of Akron, Akron, Ohio 44325, United States \\ ${ }^{\ddagger}$ Department of Arts and Sciences, The University of Akron, Akron, Ohio 44325, United States
}

\section{Supporting Information}

\begin{abstract}
Cancer cells often adapt to single-agent treatments with chemotherapeutics. Activation of alternative survival pathways is a major mechanism of drug resistance. A potential approach to block this feedback signaling is using combination treatments of a pair of drugs, although toxicity has been a limiting factor. Preclinical tumor models to identify mechanisms of drug resistance and determine low but
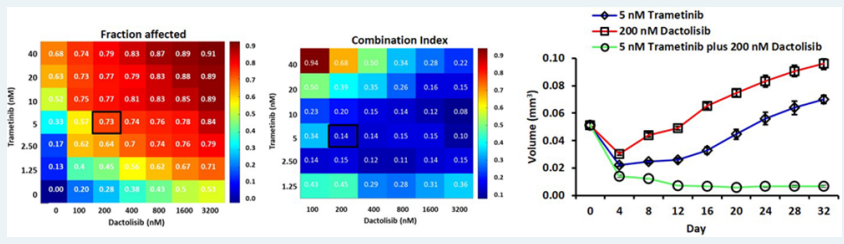
effective combination doses are critical to effectively suppress tumor growth with reduced toxicity to patients. Using our aqueous two-phase system microtechnology, we developed colorectal tumor spheroids in high-throughput and evaluated resistance of cancer cells to three mitogen-activated protein kinase inhibitors (MAPKi) in long-term cyclic treatments. Our quantitative analysis showed that the efficacy of MAPKi significantly reduced over time, leading to an increase in proliferation of HCT116 colorectal cancer cells and growth of spheroids. We established that resistance was due to feedback activation of $\mathrm{PI} 3 \mathrm{~K} / \mathrm{AKT} / \mathrm{mTOR}$ pathway. Using high-throughput, dose-dependent combinations of each MAPKi and a PI3K/mTOR inhibitor, we identified low-dose, synergistic combinations that blocked resistance to MAPKi and effectively suppressed the growth of colorectal tumor spheroids in long-term treatments. Our approach to study drug resistance offers the potential to determine high priority treatments to test in animal models.
\end{abstract}

KEYWORDS: cancer drug resistance, tumor spheroids, feedback signaling, combination treatment, cyclic treatment

A dvancements in identifying molecular drivers of cancer have shifted treatments toward precision medicine, where molecular subtype of tumors guide treatments with targeted drugs. ${ }^{1}$ Although this approach is often initially successful, the selective pressure that a targeted drug exerts can lead to resistance of cancer cells through various mechanisms. ${ }^{2,3}$ Additionally, due to the heterogeneity of cancer cells, a subpopulation of tumor cells not responsive to the targeted drug thrives to promote tumor growth. ${ }^{4}$

Mitogen-activated protein kinase (MAPK) is an oncogenic signal transduction pathway in colorectal cancer. Because this pathway is often highly deregulated, it presents an attractive therapeutic target to suppress tumor growth using inhibitors of RAF, MEK, or ERK (RAFi, MEKi, or ERKi). These inhibitors have been shown to suppress growth of colorectal tumors in vivo. ${ }^{5,6}$ Nevertheless, cancer cells often develop resistance to these inhibitors through several mechanisms: (i) Activation of alternative signaling pathways such as $\mathrm{PI} 3 \mathrm{~K} / \mathrm{AKT} / \mathrm{mTOR}$ or JAK/STAT mediates resistance to $\mathrm{MEKi}^{7}$ (ii) feedback activation of receptor tyrosine kinases (RTKs) such as epidermal growth factor receptors (EGFR, HER2, and HER3) causes resistance to RAFi and $\mathrm{MEKi}{ }^{8-10}$ (iii) continuous exposure to MEKi may lead to mutation of MEK; $^{11}$ and (iv) continuous exposure to RAFi may lead to amplification of B-RAF or other components of the MAPK pathway. ${ }^{12,13}$ To overcome drug resistance of cancer cells, combination treatments using inhibitors of RAF and MEK have been approved to treat BRAF mutant melanoma and, more recently, BRAF mutant colorectal cancer.

Over $50 \%$ of colorectal cancers have mutations in MAPK or PI3K/AKT/mTOR pathways. ${ }^{14}$ Genetic abnormalities often activate both of these pathways, resulting in a reduced response to MAPK pathway inhibition through cross-talk with the PI3K pathway. Therefore, combining inhibitors of these two pathways is a rational therapeutic approach. This approach showed promising anti-tumor effects in preclinical trials, but it was largely unsuccessful due to excessive toxicity to patients. ${ }^{5-18}$ The failure in part reflects insufficiency of preclinical models to predict drug resistance and toxicity. Dose reduction is a feasible approach to manage toxicities particularly with drugs that produce anti-tumor effects at low concentrations. However, it is difficult to test large arrays of drug combinations in standard models widely used in cancer research such as animal models. Availability of simpler preclinical models would allow identifying specific concentrations of drug combinations that generate desirable biologic effects and then advancing them to animal model tests. Physiologically relevant, three-dimensional (3D) cultures of cancer cells are promising preclinical models to address this need because they mimic the architecture and key biologic properties of tumors, facilitate understanding mechanisms of

Received: June 14, 2019

Published: July 19, 2019 
drug resistance, and help identify effective drug combinations. ${ }^{19-21}$ Because chemo-resistance often develops over time, a major barrier to model this event has been technological limitations for long-term, 3D culture of cancer cells. We recently established this capability and showed that treatment of tumor spheroids in a regimen that mimics clinical chemotherapy leads to drug resistance. ${ }^{22}$

Here, we establish the utility of this approach to identify mechanisms of drug resistance and determine low-dose effective combination treatments to maintain drug sensitivity of cancer cells. We periodically treated KRAS mutant colorectal cancer spheroids with several MAPKi and showed that effectiveness of the inhibitors to suppress proliferation of cells and growth of spheroids significantly reduced during successive treatment cycles. Molecular analysis showed that suppression of MAPK pathway was only short-lived and that repeated exposure of spheroids to MAPKi significantly activated the $\mathrm{PI} 3 \mathrm{~K} / \mathrm{AKT} / \mathrm{mTOR}$ pathway and led to proliferation of cancer cells. High-throughput screening of combinations of several MAPK and PI3K inhibitors helped identify low-dose synergistic concentrations to effectively reduce activities of both pathways and suppress tumor spheroids growth in long-term cyclic treatments. Our designdriven approach offers a useful tool for mechanistic understanding of drug responses of cancer cells and identifying lowdose, highly synergistic drug combinations to block drug resistance.

\section{MATERIALS AND METHODS}

Cell Culture and Spheroid Formation. HCT116 cells (ATCC) were cultured in McCoy's 5A medium (Sigma) supplemented with $10 \%$ fetal bovine serum (Sigma), $1 \%$ streptomycin/penicillin (Thermo Fisher Scientific), and 1\% glutamine (Thermo Fisher Scientific). Cells were cultured in a humidified incubator at $37{ }^{\circ} \mathrm{C}$ and $5 \% \mathrm{CO}_{2}$ and subcultured when they were $80-90 \%$ confluent. A $0.25 \%$ trypsin solution (Thermo Fisher Scientific) was used to dissociate cells from culture flasks. The complete growth medium was used to neutralize trypsin. The resulting cell suspension was centrifuged down at $1000 \mathrm{rpm}$ for $5 \mathrm{~min}$ at $4{ }^{\circ} \mathrm{C}$. After removing the supernatant, cells were suspended in $1 \mathrm{~mL}$ of the culture medium and counted using a hemocytometer prior to spheroid formation. Spheroids with a density of $1.5 \times 10^{4}$ cells were formed in round-bottom ultralow attachment 384-well plate (Corning) using our aqueous two-phase system (ATPS) technology, as described before. ${ }^{23,24}$ HCT116 spheroids were imaged using an inverted fluorescent microscope (Axio Observer, Zeiss) daily for 10 days to evaluate their growth.

Drug Treatments. Trametinib, SCH772984, AZ628, dactolisib, apitololisib, VS5584, PI-103, and GSK1059615 were purchased from Selleckchem. All compounds were dissolved in dimethyl sulfoxide (DMSO) except for dactolisib that was dissolved in dimethylformamide. Stock solutions were stored in $-80{ }^{\circ} \mathrm{C}$. All compounds were tested dose dependently against HCT116 spheroids. Except for SCH772984, all other compounds were prepared at concentrations of $2 \times 10^{-3} \mu \mathrm{M}, 2 \times 10^{-2} \mu \mathrm{M}, 1 \times 10^{-1} \mu \mathrm{M}, 2 \times 10^{-1}$ $\mu \mathrm{M}, 1 \times 10^{0} \mu \mathrm{M}, 2 \times 10^{0} \mu \mathrm{M}$, and $2 \times 10^{1} \mu \mathrm{M}$. SCH772984 solutions were prepared at $2 \times 10^{-4} \mu \mathrm{M}, 2 \times 10^{-3} \mu \mathrm{M}, 2 \times$ $10^{-2} \mu \mathrm{M}, 1 \times 10^{-1} \mu \mathrm{M}, 2 \times 10^{-1} \mu \mathrm{M}, 1 \times 10^{0} \mu \mathrm{M}$, and $2 \times 10^{0}$ $\mu \mathrm{M}$ concentrations. The volume of the medium in the spheroid culture plate was measured, and an equal volume from the above drug solutions was pipetted into the well of the plate. This diluted the drug concentrations in half. The DMSO content in the drug solutions did not exceed $0.1 \%$ to ensure no effect on viability of cells in spheroids. ${ }^{25}$ Vehicle control untreated spheroids were grown in drug-free cell culture medium. After 4 days of drug treatment, $10 \%(\mathrm{v} / \mathrm{v})$ Prestoblue was added to wells, and the metabolic activity of cells in spheroids was measured using a microplate reader (Synergy H1M, BioTek Instruments). ${ }^{2,26}$ The fluorescent signal from drug-treated spheroids was normalized with that from the vehicle control spheroids and used to construct dose-response curves (GraphPad Prism). A 50\% lethal dose $\left(\mathrm{LD}_{50}\right)$ value was obtained from the dose-response curve of each compound. In addition to the Prestoblue biochemical assay, phase images of spheroids were captured at the end of each treatment cycle. Diameter of each spheroid was measured in Image (NIH) and converted to volume assuming a spherical shape. Correlation analysis was performed between volume of spheroids and the corresponding fluorescent signal from the Prestoblue assay.

Cyclic Treatment and Recovery of Spheroids. Trametinib, SCH772984, and AZ628 were used to model drug resistance of HCT116 cells. HCT116 spheroids were cyclically treated with $\mathrm{LD}_{50}$ concentrations of each compound, that is, $1 \times 10^{-2} \mu \mathrm{M}$ trametinib, $1.5 \times 10^{-1} \mu \mathrm{M} \mathrm{SCH} 772984$, and $1 \times 10^{0} \mu \mathrm{M}$ AZ628. Each experiment included four cycles of treatment, each cycle followed by a recovery phase. The treatments were designated as $\mathrm{T} 1, \mathrm{~T} 2, \mathrm{~T} 3$, and $\mathrm{T} 4$, whereas the recoveries were denoted as R1, R2, R3, and R4. Each treatment and recovery phase lasted 4 days. Each treatment phase included drug addition to spheroids at the beginning only. At the end of each treatment phase, the drug-containing culture medium was thoroughly removed from the microwells and replaced with fresh medium. The same concentration of each inhibitor was used during all treatment rounds. To quantify resistance of HCT116 spheroids to each inhibitor, a growth rate metric $\left(k_{c}\right)$ was defined as the difference in the size of spheroids after and before each treatment.

Combination Treatments of Spheroids. Trametinib, SCH772984, and AZ628 were used in combination with dactolisib, each inhibitor at six different concentrations. These concentrations were multiples $(0.125,0.25,0.5,1,2$, and 4$)$ of $\mathrm{LD}_{50}$ of each compound. For combination experiments, solutions of $4 \times$ concentrations of multiples of $\mathrm{LD}_{50}$ of each compound were prepared (i.e., 0.5, 1, 2, 4, 8, and 16 times). From these solutions, $20 \mu \mathrm{L}$ of each MAPKi solution and 20 $\mu \mathrm{L}$ of dactolisib solution were added to each well containing a spheroid in $40 \mu \mathrm{L}$ of cell culture medium to dilute each compound four folds. As each concentration of MAPKi was combined with six different concentrations of dactolisib, this resulted in a $6 \times 6$ matrix of concentration pairs for each combination treatment. In addition, single-agent treatments with MAPKi and dactolisib were performed to compare with the combination treatments. Solutions of $2 \times$ concentration (i.e., $0.25,0.5,1,2,4$, and 8 times) were prepared, and $40 \mu \mathrm{L}$ of each solution was added to each microwell containing a spheroid in $40 \mu \mathrm{L}$ of cell culture medium. Vehicle control spheroids were cultured in cell culture medium. Treatments were done for 4 days, and viability of cells was quantified using a Prestoblue assay. The fraction of cells affected by each treatment was calculated as $(1-$ viability $)$. A synergy analysis was performed using the Chou and Talalay method. ${ }^{26,27}$ The analysis generated 36 combination indices (CI) for the 36 combination concentrations from a pair of inhibitors. In 
addition, images of spheroids were captured to quantify the size of spheroids as a measure of effect of drug treatments.

Western Blot Experiments. Western blot analysis with spheroids was performed according to our established protocol. ${ }^{25,28}$ Solutions of primary antibodies for phosphop44/42 MAPK (Erk1/2), p44/42 MAPK (Erk1/2), phosphoAKT (Ser473), and AKT (pan) (C67E7) were prepared at concentrations recommended by the manufacturer, Cell Signaling Technology. Membranes were incubated overnight at $4{ }^{\circ} \mathrm{C}$ with primary antibody solutions. After repeated washing, membranes were incubated with a horseradish peroxidase (HRP)-conjugated secondary antibody for $1 \mathrm{~h}$, followed by repeated washing. Detection was carried out using an ECL chemiluminescence detection kit (GE Healthcare) and FluorChem E imaging system (ProteinSimple).

q-PCR Experiments. Gene expression analysis was done after T1, R1, T3, and R3. All fold changes values were expressed relative to that after T1. Spheroids were lysed using a Total RNA Kit (TRK) lysis buffer (Omega Biotek), and the lysate was homogenized by passing it through homogenizer mini columns (Omega Biotek). Total RNA was obtained using an RNA isolation kit (Omega Biotek). After removing DNA using RNase-free DNase (Omega Biotek), purity and concentration of isolated RNA were assessed using optical density (OD) 260/280 spectrophotometry (Synergy H1M, Biotek Instruments). cDNA was synthesized from $1 \mu \mathrm{g}$ of total RNA using random hexamer primers (Roche). Real-time qPCR was performed in a LightCycler 480 instrument II using a SYBR Green Master Mix (Roche). After combining $50 \mathrm{ng}$ of cDNA with the primer and the SYBR Green Master Mix to a final volume of $15 \mu \mathrm{L}$, the reactions were incubated at $95{ }^{\circ} \mathrm{C}$ for $5 \mathrm{~min}$ followed by 45 cycles of amplification, that is, at 95 ${ }^{\circ} \mathrm{C}$ for $10 \mathrm{~s}$, at $60{ }^{\circ} \mathrm{C}$ for $10 \mathrm{~s}$, and at $72{ }^{\circ} \mathrm{C}$ for $10 \mathrm{~s}$. The primer sequences for the genes are listed in Table S1 of Supporting Information. Expression levels of mRNA for different proliferation gene markers were calculated relative to $\beta$-actin and hypoxanthine phosphoribosyltransferase (HPRT) using the $\Delta \Delta \mathrm{C}_{\mathrm{t}}$ method. The fold change in mRNA expression was determined according to the $2^{-\Delta \Delta \mathrm{C}_{\mathrm{t}}}$ method. ${ }^{29,30}$ Statistical analysis was performed using a Student's $t$-test in Microsoft Excel software.

Confocal Microscopy. Prior to forming spheroids, HCT116 cells were stained with $2 \mu \mathrm{M}$ of Calcein AM dye (Thermo Fisher Scientific) when cells were in a monolayer culture. Confocal microscopy of spheroids was performed using Nikon A1 confocal system with $10 \times$ objective. FITC filter was used to capture image stacks with a z-spacing of 20 $\mu \mathrm{m}$. NIS Elements software was used for image acquisition, and ImageJ (NIH) was used for analysis and 3D reconstruction.

Statistical Analysis. Student's t-test between cyclic treatments and Pearson's correlation coefficient between volume of spheroids and the corresponding fluorescent signal from Prestoblue assay were performed in Microsoft Excel.

\section{RESULTS AND DISCUSSION}

Microprinting of Tumor Spheroids and Their Growth Analysis. The aqueous DEX phase nanodrop containing cancer cells stably remained immiscible from the PEG phase solution. $^{31}$ This facilitated aggregation of cancer cells into a spheroid within $48 \mathrm{~h}$ (Figure 1a). The ATPS environment allowed a free diffusional influx of nutrients into the drop phase to nourish the cells and efflux of waste products of cells into

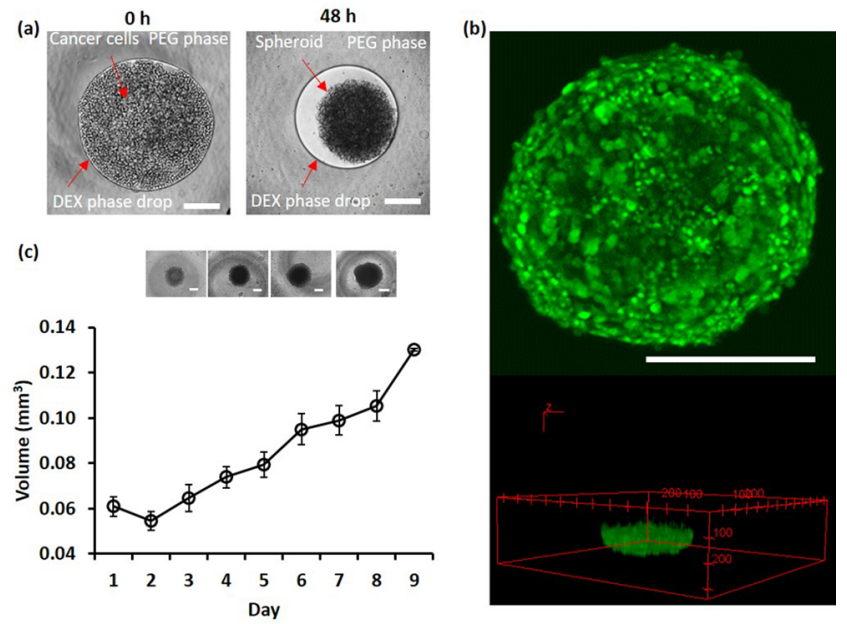

Figure 1. Formation and growth of tumor spheroids. (a) A $0.3 \mu \mathrm{L}$ drop of the aqueous DEX phase containing HCT116 cancer cells immersed in the aqueous PEG phase settles to the well bottom. Cancer cells remain within the DEX drop and form a spheroid. (b) Confocal image of spheroid shows fully viable cells and a 3D z-stack reconstruction of a portion of a spheroid. (c) Size of HCT116 spheroids increases during incubation indicating cell proliferation. Inset images from left to right represent spheroids on days 2, 4, 6, and 8. Error bars represent standard error from the mean $(n=14)$. Scale bar is $200 \mu \mathrm{m}$ in panels $(\mathrm{a}-\mathrm{c})$.

the immersion phase. The ATPS microprinting approach provided a mild environment for cells to form spheroids of fully viable cells. ${ }^{32,33}$ Medium exchange every $48 \mathrm{~h}$ provided fresh nutrients and removed waste products of cells. This also significantly reduced concentrations of the polymers and resulted in a single medium phase. Thus, ATPS was solely used to quickly and conveniently generate spheroids. We have shown that at the same cell density, the ATPS approach generates spheroids that are $\sim 30 \%$ more compact than those from the ULA plate method. ${ }^{34}$ Additionally, we have adapted the ATPS technology to robotic liquid handling to form large quantities of consistently sized spheroids in 384-microwell plates. ${ }^{35}$ With a density of $1.5 \times 10^{4}$ HCT 116 cells per DEX drop, a variation of $<5.5 \%$ from an average diameter of $468 \mu \mathrm{m}$ resulted. HCT116 spheroids had a distinct boundary and a round and compact morphology, indicated by both phase and confocal imaging (Figure 1a,b). Our morphological image analysis showed an increase in the average diameter of spheroids from $468 \mu \mathrm{m}$ on day 1 to $650 \mu \mathrm{m}$ on day 9 , that is, $\sim 38 \%$ increase in the diameter of spheroids. Approximating the spheroids as spherical clusters, this corresponds to a 2.16fold volume increase, indicating proliferation of cancer cells within the spheroids (Figure 1c).

Dose-Response to Molecular Inhibitors. KRAS and PIK3CA mutations in HCT116 cells activate oncogenic MAPK and PI3K/AKT/mTOR pathways. ${ }^{36}$ Therefore, we aimed to determine the effect of blocking signaling through these pathways in HCT116 spheroids using a set of molecular inhibitors including trametinib, SCH772984, and AZ628 against the MAPK pathway and dactolisib, apitolisib, VS5584, PI-103, and GSK1059615 against the PI3K/AKT/ mTOR pathway. The MAPKi dose dependently reduced cell viability in spheroids (Figure 2a). Trametinib was the most effective inhibitor with an $\mathrm{LD}_{50}$ of $10 \mathrm{nM}$. Above $100 \mathrm{nM}$, trametinib treatment led to fluffiness or disintegration of 
(a)

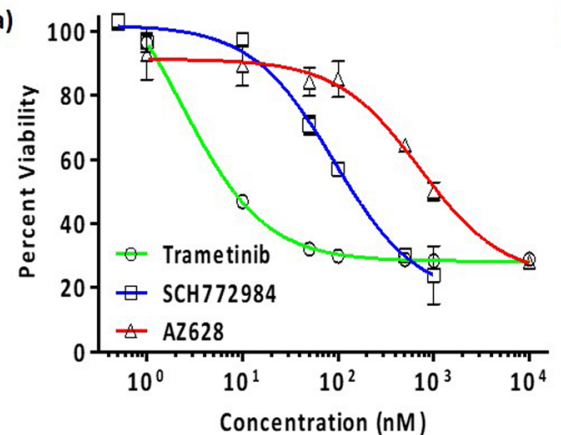

(b)

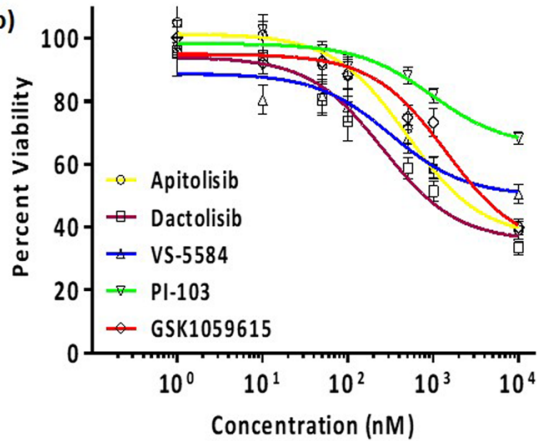

(c)

\begin{tabular}{lll}
\hline Drug & Target & LD $_{50}(\boldsymbol{\mu M})$ \\
Trametinib & MEK & 0.01 \\
SCH772984 & ERK & 0.15 \\
AZ628 & RAF & 1 \\
Apitolisib & PI3K & 2 \\
Dactolisib & PI3K/mTOR & 0.8 \\
VS-5584 & PI3K/mTOR & 7 \\
PI-103 & PI3K & - \\
GSK1059615 & PI3K/mTOR & 2 \\
\hline
\end{tabular}

Figure 2. Responses of spheroids to MAPK and PI3K inhibitors. Dose-responses of HCT116 spheroids to inhibitors of (a) MAPK pathway and (b) PI3K pathway. (c) The list of molecular inhibitors used, their targets, and $\mathrm{LD}_{50}$ values against $\mathrm{HCT} 116$ spheroids. The - indicates an $\mathrm{LD}_{50}$ value could not be obtained.

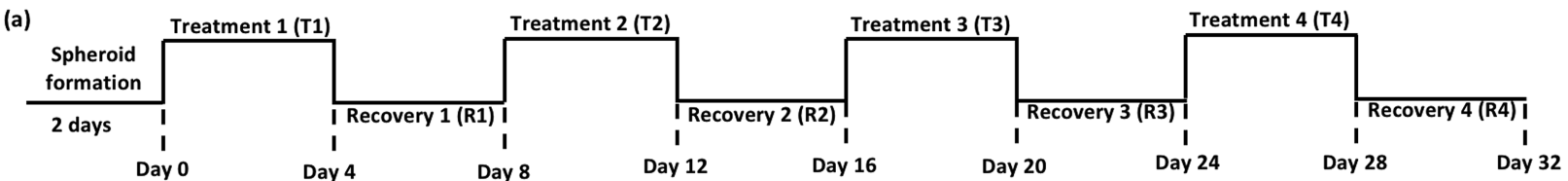

(b)

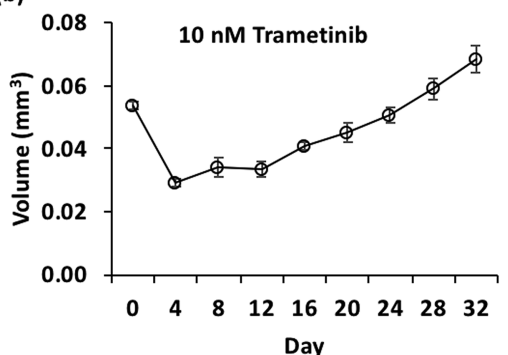

(e)

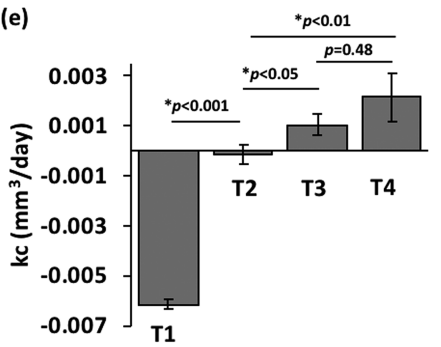

(c)

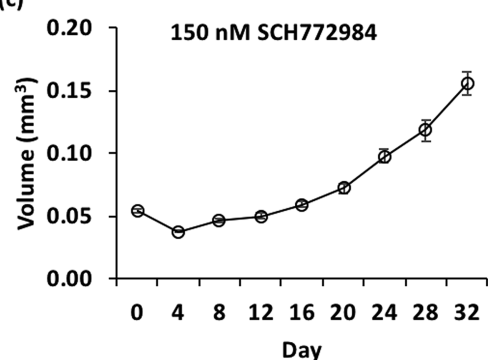

(f)

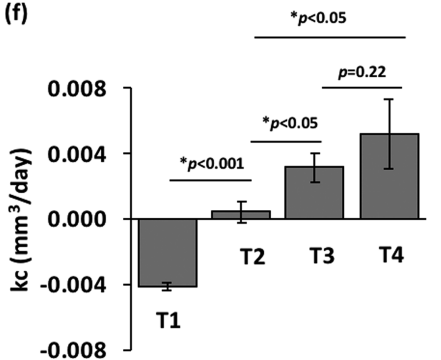

(d) 0.16$] \quad 1 \mu \mathrm{M}$ AZ628

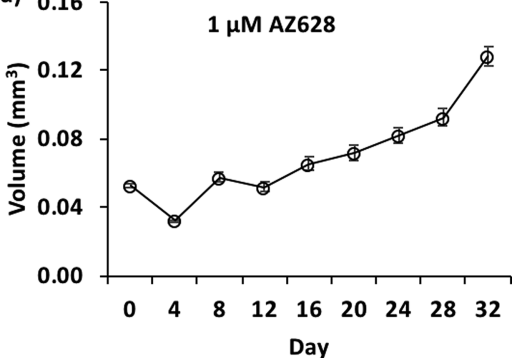

(g)

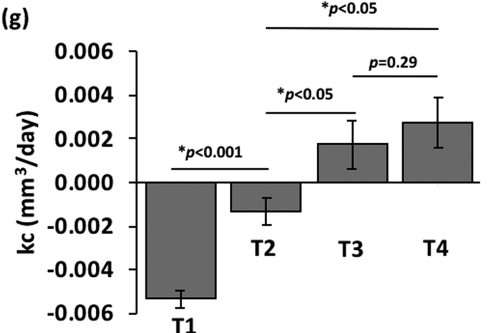

Figure 3. Cyclic drug treatment and recovery of tumor spheroids. (a) HCT116 spheroids were cyclically treated with inhibitors of MEK (0.01 $\mu \mathrm{M}$ trametinib), ERK (0.15 $\mu \mathrm{M}$ SCH772984), and RAF (1 $\mu \mathrm{M} \mathrm{AZ628).} \mathrm{(b-d)} \mathrm{Kinetics} \mathrm{of} \mathrm{spheroids} \mathrm{growth} \mathrm{during} \mathrm{cyclical} \mathrm{treatment} \mathrm{and} \mathrm{recovery.}$ Each data point in the line graph is an average of 32 replicates. $(\mathrm{e}-\mathrm{g})$ Growth rate $\left(k_{\mathrm{c}}\right)$ of HCT116 spheroids during four treatment rounds with (e) trametinib, (f) SCH772984, and (g) AZ628. $n=14$ and $*$ denotes statistically significant differences in the growth rates between treatment rounds. Error bars in panels $(\mathrm{b}-\mathrm{g})$ represent the standard error from a mean value.

spheroids. SCH772984 was effective above $100 \mathrm{nM}$ concentrations and resulted in an $\mathrm{LD}_{50}$ of $150 \mathrm{nM}$, whereas AZ628 treatment significantly reduced cell viability at low micromolar concentrations and gave an $\mathrm{LD}_{50}$ of $1 \mu \mathrm{M}$. Additionally, the $\mathrm{PI} 3 \mathrm{~K} / \mathrm{AKT} / \mathrm{mTOR}$ inhibitors reduced viability of HCT116 cells dose dependently but at significantly larger, mainly micromolar concentrations than the MAPKi did (Figure 2b,c).

The volume of spheroids treated with the MAPKi showed a linear correlation with the cellular metabolic activity from a Prestoblue assay. The goodness-of-fit parameter $\left(R^{2}\right)$ values were $0.997,0.973$, and 0.887 for treatments with trametinib, SCH772984, and AZ628, respectively. The corresponding Pearson's correlation coefficients between dose-dependent reduction in the volume of spheroids and metabolic activity from the biochemical analysis were 0.99, 0.98, and 0.94. The volume of spheroids treated with these PI3K pathway inhibitors also linearly correlated with the fluorescent signal obtained using Prestoblue assay. This analysis validated that morphology-based size analysis of colorectal tumor spheroids treated with kinase molecular inhibitors reliably predicts the effect of treatments with the molecular inhibitors. ${ }^{34}$

Resistance of Colorectal Spheroids to Cyclic Treatments with MAPKi. Our screening above showed that HCT116 spheroids were more sensitive to inhibition of the MAPK pathway than targeting of the PI3K/AKT/mTOR pathway, suggesting blocking of the MAPK pathway as a strategy to inhibit proliferation of cells in spheroids. We evaluated responses of HCT116 spheroids to long-term, cyclic 
treatment and recovery with the $\mathrm{LD}_{50}$ concentrations of the three MAPKi (Figure 3a). We used this regimen to mimic the cyclic chemotherapy of patients. As expected, the compounds potently inhibited proliferation of cancer cells during the first treatment round (T1) (Figure $3 \mathrm{~b}-\mathrm{d}$ ). The size of spheroids treated with trametinib, SCH772984, and AZ628 decreased by $1.80-, 1.42-$, and 1.67-fold, respectively. However, after the first recovery round (R1), the inhibitors were significantly less effective during treatment T2. Despite repeated treatments, the HCT116 spheroids grew larger. At the end of the 32-day treatment and recovery, spheroids treated with trametinib, SCH772984, and AZ628 were 2.34-, 4.14-, and 4.04-fold larger than those at the end of treatment $\mathrm{T} 1$, respectively.

Furthermore, we used a growth rate metric $\left(k_{\mathrm{c}}\right)$ to quantify the effects of treatments (Figure $3 \mathrm{e}-\mathrm{g}$ ). Although $k_{\mathrm{c}}$ for HCT116 spheroids significantly reduced during $\mathrm{T} 1$, it increased during the subsequent rounds of treatment. The $k_{c}$ values of spheroids from $\mathrm{T} 1$ to $\mathrm{T} 4$ significantly increased from -0.0061 to $0.0021 \mathrm{~mm}^{3} /$ day for trametinib treatment, from -0.0040 to $0.0052 \mathrm{~mm}^{3} /$ day for SCH772984 treatment, and from -0.0050 to $0.00271 \mathrm{~mm}^{3} /$ day for AZ628 treatment. The significant decrease in the efficacy of the MAPKi indicates that cancer cells in spheroids quickly adapt to the inhibition of different proteins (RAF, MEK, and ERK) in this pathway. Interestingly, our drug resistance model reliably emulated several in vivo studies that showed cyclic treatments of tumor xenografts with MEK1/2 inhibitors did not reduce the tumor size, necessitating other treatments. ${ }^{37,38}$

Next, we aimed to study whether increasing growth of cancer cells during cyclic inhibition of MAPK pathway could be detected at a gene level. We selected 10 prominent genes involved in cell cycle and proliferation based on a literature review (Table S1). Our rationale for this selection was that these genes are activated by transcriptional factors downstream of MAPK and PI3K signaling pathways, which are drivers of the growth of HCT 116 cells in tumor spheroids. We compared the expression of each gene after the first and third cycles of treatment and recovery, that is, T1, R1, T3, and R3. Expression of each gene after R1, T3, and R3 is shown in Figure 4 as a fold change relative to that after T1. Most of the genes showed a significant upregulation during the cycles of treatment and recovery irrespective of the inhibitor used. With all three inhibitors, the largest expression of proliferation genes almost always occurred during recovery phases, especially the R3 phase, with only few exceptions. In addition, during T3, expression levels of some of the genes slightly reduced compared to the $\mathrm{R} 1$ phase, indicating a transient response to the treatments. However, this reduction was not significant in most cases, which is also evident from the growth of spheroids during T3 (Figure $3 \mathrm{~b}-\mathrm{g}$ ). Overall, relatively higher expression levels of proliferation genes in $\mathrm{R} 3$ than in $\mathrm{R} 1$ and in $\mathrm{T} 3$ than in $\mathrm{T} 1$ corroborate our findings of increased resistance of cancer cells to MAPKi with successive cycles of drug exposure.

Mechanism of Drug Resistance. Next, we investigated the molecular basis of increasing resistance of cancer cells to cyclic single-agent treatments with MAPKi. Studies have shown that inhibition of MEK1/2 can activate the PI3K/ $\mathrm{AKT} / \mathrm{mTOR}$ pathway due to extensive cross-talk between the two pathways. ${ }^{39,40}$ Thus, we performed a Western blot analysis for both ERK1/2 and AKT in HCT116 spheroids cyclically treated with the three MAPKi (Figure 5a). All three inhibitors significantly reduced $p E R K 1 / 2$ levels. Consistent with the phenotypic analysis result during T1 (Figure 3), trametinib
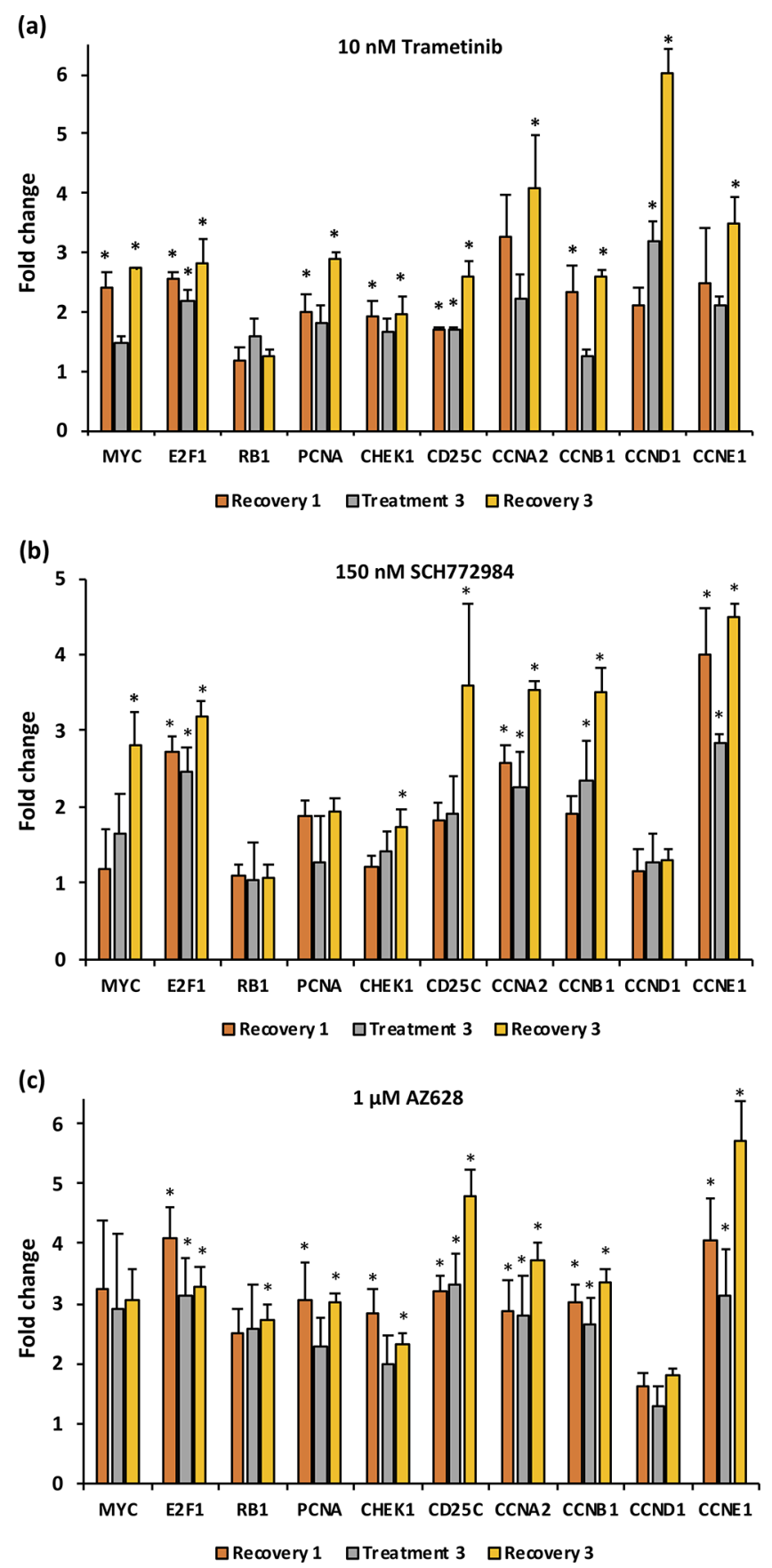

Figure 4. Fold change in expression of proliferation genes in tumor spheroids during cyclic treatment and recovery with MEKi. The bar graphs show the fold change values of 10 prominent proliferation genes after treatments with (a) $10 \mathrm{nM}$ trametinib, (b) $150 \mathrm{nM}$ SCH772984, and (c) $1 \mu \mathrm{M}$ AZ628. The fold change values after recovery 1 , treatment 3 , and recovery 3 are relative to treatment 1 . * denotes $p<0.05$ when compared with treatment 1 .

most effectively downregulated ERK $1 / 2$ activity by $\sim 85 \%$ (Figure 5b). This was followed by SCH772984 and AZ628 treatments that reduced $p E R K 1 / 2$ levels by $56 \%$ and $25 \%$, respectively (Figure 5b). However, trametinib, SCH772984, and AZ628 treatments significantly increased pAKT levels by $37 \%$, $52 \%$, and $46 \%$ of the vehicle control spheroids, respectively (Figure 5a,c). This established that the targeting MAPK pathway at different levels (RAF, MEK, and ERK) 

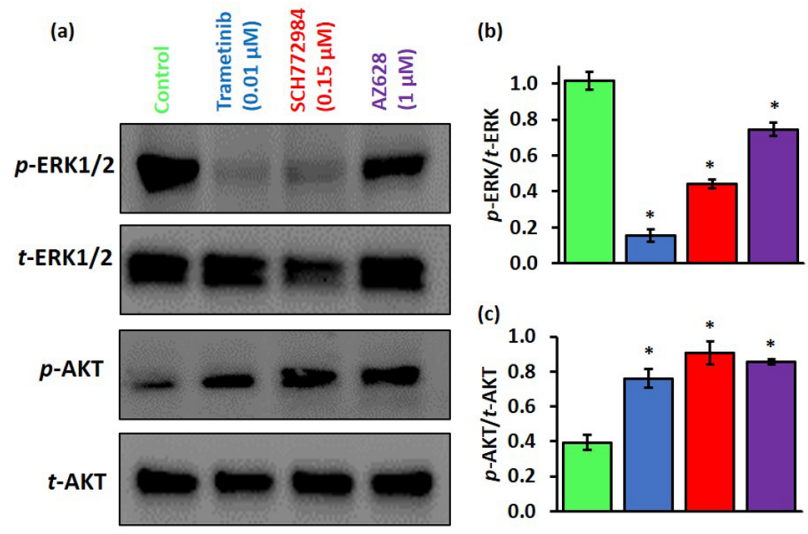

Figure 5. Activity levels of ERK $1 / 2$ and AKT proteins in tumor spheroids treated with inhibitors of MAPK pathway. (a) Western blots for phosphorylated and total levels of ERK1/2 and AKT at the end of treatment 1 (T1). (b) Quantified results of $p$-ERK/t-ERK in vehicle control and treated spheroids. (c) Quantified levels of $p$-AKT/ $t$-AKT in vehicle control and treated spheroids. Results are shown as mean \pm standard error. Each Western blot experiment was repeated twice. $*$ denotes $p<0.05$ when comparing each treatment and the vehicle control.

results in feedback activation of $\mathrm{PI} 3 \mathrm{~K} / \mathrm{AKT} / \mathrm{mTOR}$ pathway. $^{41,42}$

Additionally, we found that several proliferation genes downstream of AKT such as CCNA1, PCNA, and CCND1 were upregulated by MAPKi treatment. For example, CDC25C that encodes cyclin-dependent kinase (CDK2) and CCNA2 that encodes cyclin A mediate formation of the PI3K/ AKT-dependent CDK2/cyclin complex, which is responsible for cell proliferation. ${ }^{41,42}$ Furthermore, AKT-dependent phosphorylation of $\mathrm{p} 21$ prevents a complex formation with proliferating cell nuclear antigen (PCNA), which inhibits DNA replication. ${ }^{43}$ Increase in PCNA during cyclic trametinib and AZ628 treatments indicates AKT-mediated release of PCNA for DNA replication and growth of tumor spheroids. We also suspect that pathways other than $\mathrm{PI} 3 \mathrm{~K} / \mathrm{AKT} / \mathrm{mTOR}$ could drive growth of tumor spheroids. Our previous study showed that the increased growth of spheroids during successive treatment/recovery cycles and development of resistance to MEKi treatments is in part due to incomplete suppression of ERK activity. ${ }^{22}$ We identified several cell cycle regulating genes downstream of ERK such as CCND1, CDC25C, and MYC that were significantly upregulated during R1, T3, and R3, indicating that incomplete suppression of ERK by MEKi treatments also leads to cell proliferation and growth of spheroids.

Combination Treatments To Suppress Resistance. Next, we evaluated the potential of a combination treatment strategy to block resistance of HCT116 spheroids to MAPKi treatments. We combined trametinib, SCH772984, and AZ628 with a PI3K/mTOR inhibitor, dactolisib, which had the smallest $\mathrm{LD}_{50}$ value among the $\mathrm{PI} 3 \mathrm{~K} / \mathrm{AKT} / \mathrm{mTOR}$ inhibitors used against HCT116 spheroids (Figure 2b,c). Each combination treatment included 36 pairs of concentrations of a pair of inhibitors. We computed the fraction of cells affected $\mathrm{Fa}$ by treatments. Figure $6 \mathrm{a}-\mathrm{c}$ shows the results for combination treatments of trametinib/dactolisib, SCH772984/dactolisib, and AZ628/dactolisib pairs, respectively, as well as a single-agent treatment with each inhibitor. The Fa values increased at higher concentration pairs, represented by a darker shade of red in the heatmaps. Combination treatments were also significantly more effective than respective single-agent treatments at similar concentrations. To elucidate whether effects of drug combinations were synergistic, we computed a CI for each pair. Using COMPUSYN software, we performed a synergy analysis to generate a CI value for each concentration pair. CI $<1$ indicates synergism and the synergy level increases as CI approaches zero. ${ }^{26,27}$ All 36 concentration pairs resulted in CI $<1$, indicating synergistic interactions between each of the MAPKi and dactolisib and the potential of blocking these two pathways to block cancer cell proliferation and survival. The CI values ranged from 0.11 to 0.94 for trametinib/dactolisib, 0.05 to 0.48 for SCH772984/dactolisib, and 0.09 to 0.85 for AZ628/dactolisib (Figure 6d-f). In addition, we captured the morphology of spheroids in each experiment. The combination pairs, especially at higher concentrations, partially or completely disintegrated spheroids, suggesting toxicity to cancer cells. ${ }^{25}$ From each combination treatment, the pairs of concentrations that disintegrated the spheroids are highlighted in red in Table S2.

To study inhibitory effects of combination treatments on MAPK and PI3K/AKT pathways, we performed Western blotting of spheroids following treatment T1. Treatments included $5 \mathrm{nM} / 200 \mathrm{nM}$ trametinib/dactolisib, $75 \mathrm{nM} / 400 \mathrm{nM}$ SCH772984/dactolisib, and $500 \mathrm{nM} / 800 \mathrm{nM} \mathrm{AZ628/}$ dactolisib. This selection reflects strong synergistic growth inhibitory effects $(\mathrm{CI}=0.1-0.3)$ against HCT116 spheroids at sufficiently low, nanomolar concentrations that did not disintegrate the spheroids (Figure $6 \mathrm{~d}-\mathrm{f}$ and Table S2). We also used a single-agent treatment with each inhibitor to compare with combination treatments. As expected, singleagent treatments with the MAPKi significantly downregulated $p$-ERK levels (top lanes in Figure $6 \mathrm{~g}-\mathrm{i}$ ), leading to growth inhibition of HCT116 spheroids. Nevertheless, AKT activity significantly increased (third lanes in Figure $6 \mathrm{~g}-\mathrm{i}$ ). Interestingly, the $p$-AKT level did not significantly reduce after singleagent dactolisib treatment. Dactolisib is a potent inhibitor of PI3K and mTOR. It is likely that the growth inhibitory effect of dactolisib is through mTOR inhibition. ${ }^{44}$ For example, it was shown that downregulation of substrates of mTORC1 such as pS6 and p4EBP1 inhibited growth of HCT116 cells. ${ }^{45}$ Dactolisib also inhibits DNA-PK and class II PI3Ks, which may contribute to its growth inhibition of HCT116 cells. ${ }^{46}$ Unlike significant activation of AKT in response to inhibition of MAPK pathway, there was no significant increase in the level of $p$-ERK $1 / 2$ following dactolisib treatment of HCT116 spheroids. This implies an absence of feedback activation of MAPK pathway by PI3K/AKT pathway inhibition in these cells.

Combined inhibitors of MAPK and PI3K pathways significantly reduced active levels of both ERK1/2 and AKT proteins. Trametinib/dactolisib, SCH772984/dactolisib, and AZ628/dactolisib pairs reduced $p$-ERK1/2 levels by 70\%, 53\%, and $67 \%$ of the vehicle control, respectively (Figure $6 \mathrm{~g}-\mathrm{i}$ ). Each combination treatment also further reduced $p$ ERK $1 / 2$ levels than the single-agent treatment with the corresponding MAPKi. The largest reduction of $60 \%$ was with the AZ628/ dactolisib pair compared to AZ628 alone. This was followed by the $\mathrm{SCH} 772984 /$ dactolisib pair that reduced the $p \mathrm{ERK} 1 / 2$ level 24\% more than that when SCH772984 was used alone. The trametinib/dactolisib pair also reduced the $p$ ERK1/2 level $13 \%$ more than that with trametinib treatment only. We note 
(a)

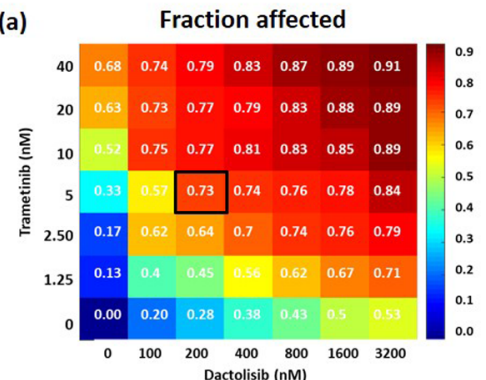

(b)

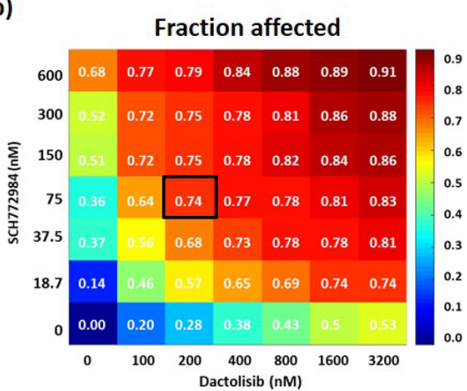

(c)

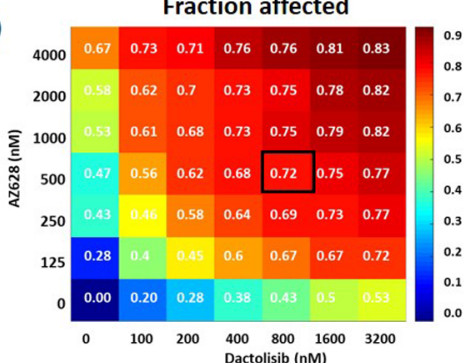

(d)

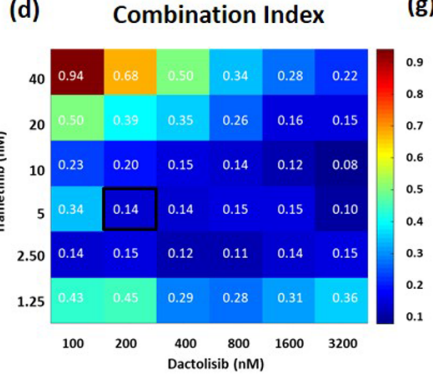

(g)
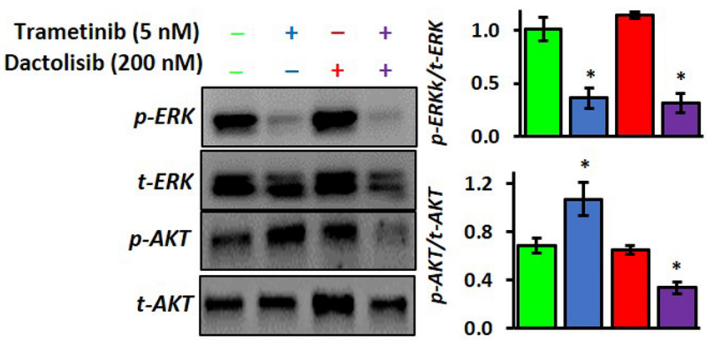

(h)

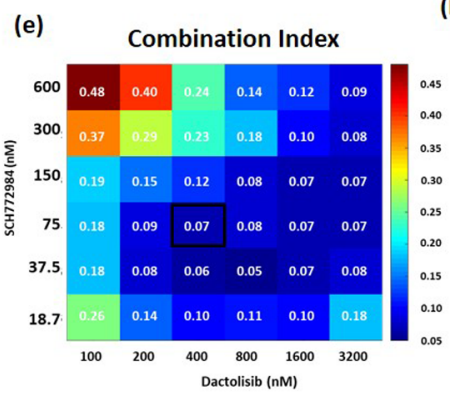

SCH772984 (75 nM) - + - + Dactolisib (400 nM) - _ + +
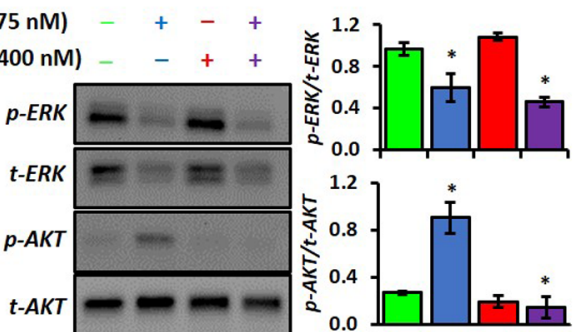

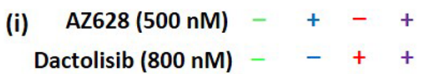

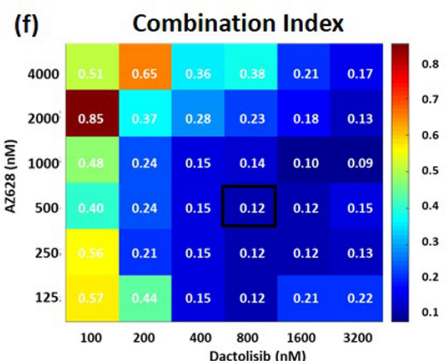

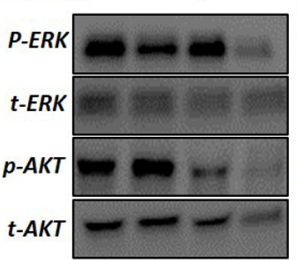

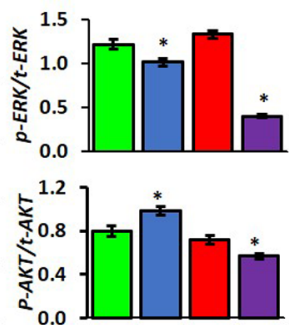

Figure 6. Combination treatments of spheroids with inhibitors of MAPK and PI3K pathways. Heatmap plots representing fraction of cells affected (Fa) by single-agent and combination treatments of (a) trametinib/dactolisib, (b) SCH772984/dactolisib, and (c) AZ628/dactolisib. Heatmaps representing combination indices (CI) following combination treatments of spheroids with (d) trametinib/dactolisib, (e) SCH772984/dactolisib, and (f) AZ628/dactolisib. The combination concentrations resulting in greater cell death (larger Fa values) and higher synergy (smaller CI values) are boxed in the heatmaps of panels $(a-f)$. This pair of concentrations from each pair of inhibitors was selected for Western blots of active and total ERK1/2 and AKT shown in panels $(\mathrm{g}-\mathrm{i})$. Bar graphs represent quantified $p$-ERK/t-ERK and $p$-AKT/t-AKT levels for single-agent and combination treatments. Each Western blot experiment was repeated twice. Results are shown as mean \pm standard error. ${ }^{*} p<0.05$ denotes comparing single-agent MAPKi treatments with the vehicle control and also combination treatments with the corresponding single-agent MAPKi treatments.

that this small decrease in the $p E R K 1 / 2$ level by the trametinib/dactolisib pair is because trametinib treatment alone was very effective against ERK1/2 activity. Most importantly, combination treatments were highly effective against feedback signaling of kinase pathways. Compared to single-agent treatments with trametinib, SCH772984, and AZ628 that led to activation of $\mathrm{PI} 3 \mathrm{~K} / \mathrm{AKT} / \mathrm{mTOR}$ pathway, the compounds simultaneously administrated with dactolisib downregulated $p$ AKT levels 3.17, 6.32, and 1.74 folds, respectively. In all three combination treatments, the $p A K T$ levels were significantly lower than that of the vehicle control spheroids. These results, which are consistent with several animal model studies, ${ }^{37,45,47}$ indicate the need for simultaneous blocking of compensatory signaling pathways that mediate resistance to single-agent therapies. Importantly, we identified growth inhibition of tumor spheroids using synergistic nanomolar concentrations of compounds for the subsequent long-term drug combination studies below.

Long-Term, Cyclic Combination Treatments. We established that combinations of MAPKi and dactolisib act synergistically on HCT116 spheroids in short-term, 4-day experiments. To evaluate the effectiveness of this strategy during long-term cyclic treatments (Figure 3a), we used trametinib/dactolisb against HCT116 spheroids. We selected 5 $\mathrm{nM}$ trametinib and $200 \mathrm{nM}$ dactolisib concentrations that resulted in $\mathrm{Fa}=0.73$ and a strong synergism with $\mathrm{CI}=0.14$ (Figure 6a,d). In parallel, we performed single-agent treatments at the same concentration of each inhibitor used in the combination treatment. Unlike trametinib or dactolisib treatments alone, their combination significantly and effectively suppressed the growth of spheroids over a 32-day period (Figure 7a). To quantitatively compare the combination and single-agent treatments, we calculated the growth rate of spheroids. The average values of $k_{\mathrm{c}}$ for $5 \mathrm{nM}$ trametinib, 200 $\mathrm{nM}$ dactolisib, and their combination were $0.0006,0.0014$, and $-0.00135 \mathrm{~mm}^{3} /$ day, respectively. The positive $k_{\mathrm{c}}$ values indicate that the efficacy of trametinib and dactolisib treatments diminished over time. On the other hand, the combination treatment resulted in a negative $k_{c}$ value, indicating continuous growth suppression of tumor spheroids.

Our molecular analysis corroborated this result. We compared active AKT and ERK1/2 levels after T1 and T3 


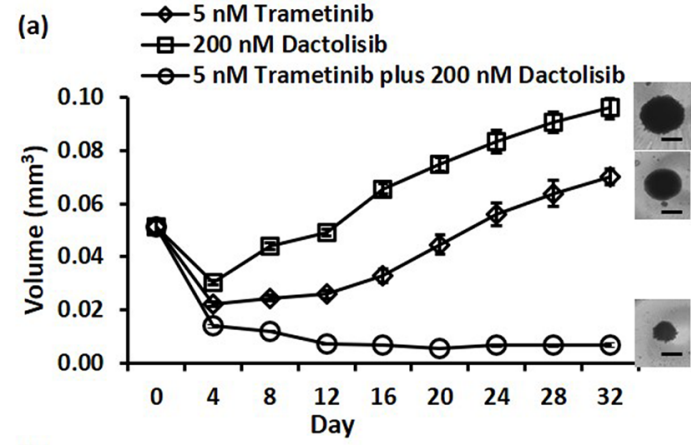

(b)

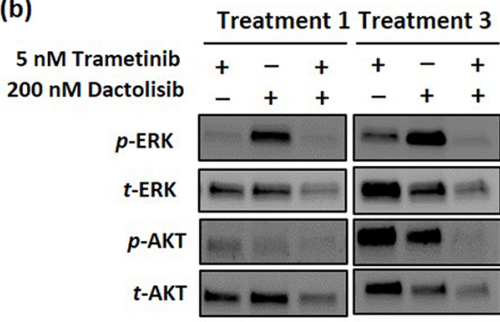

(c)

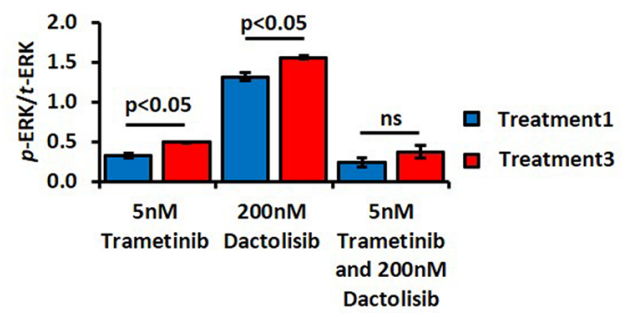

(d)

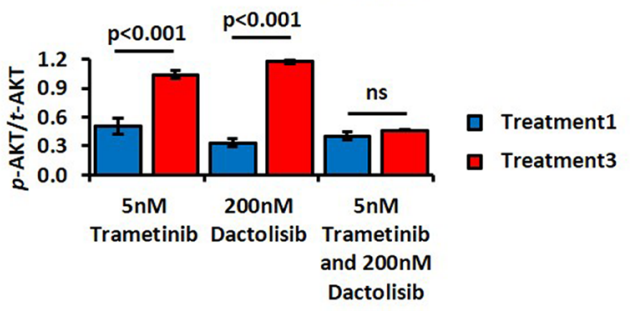

Figure 7. Long-term single-agent and combination treatment/ recovery of spheroids. (a) Size of spheroids under cyclic singleagent and combination treatments with trametinib and dactolisib. Each data point in the graph is an average of 32 replicates. Error bars represent standard error from the mean. Inset images show spheroids from single-agent and combination treatments at the end of 32 days of culture. Scale bar is $250 \mu \mathrm{m}$. (b) Representative Western blots of active and total ERK1/2 and AKT for single-agent and combination treatments at the end of treatment 1 (T1) and treatment 3 (T3). Quantified levels of (c) $p$-ERK/t-ERK and (d) $p$-AKT $/ t$-AKT. Each Western blot experiment was repeated twice. Results are shown as mean \pm standard error.

for single-agent and combination treatments. Although trametinib treatment alone reduced ERK1/2 activity during $\mathrm{T} 1$, which caused a significant shrinking of the spheroids, it was ineffective during T3, and the ERK1/2 activity significantly increased (Figure $7 \mathrm{~b}, \mathrm{c}$ ). The reduced effectiveness of the single-agent trametinib treatment is also consistent with the upregulation of proliferation genes downstream of ERK such as MYC, CDC25, and CCND1 (Figure 4a). ${ }^{48}$ In addition to the $p$-ERK increase during T3, trametinib-induced activation of $p$ AKT persisted (Figure $7 \mathrm{~b}, \mathrm{~d}$ ), resulting in growth of HCT116 spheroids (Figure 7a). Dactolisib treatment alone did not suppress $p$-AKT levels, and despite the treatment, the AKT activity increased from $\mathrm{T} 1$ to T3 (Figure $7 \mathrm{~b}, \mathrm{~d}$ ). The reduced effect of dactolisib used alone is also consistent with the upregulation of proliferation genes downstream of AKT such as CCNA2, PCNA, and CCND1. ${ }^{49}$ Therefore, elevated activities of AKT and ERK1/2 account for growth of spheroids over the long-term, single-agent cyclic treatments. On the other hand, the trametinib/dactolisib combination maintained the $p$-ERK and $p$-AKT levels low (Figure 7c,d). Thus, inhibition of both pathways was critical to suppress the growth of cancer cells. Anti-tumor effects of combinations of MEK and PI3K inhibitors in animal model studies support our results, demonstrating the potential of tumor spheroid model in cyclic treatments to identify synergistic drug pairs against cancer cell growth. ${ }^{47,50-52}$

\section{CONCLUSION}

We established that colorectal cancer cells in a spheroid culture under cyclic, single-agent treatments with MAPK pathway inhibitors develop resistance by activation of the PI3K feedback signaling. Using a rational-design strategy guided by molecular analysis of drug resistance, we combined inhibitors of initially active (MAPK) and feedback activated (PI3K) pathways to overcome drug resistance. Our systematic, highthroughput drug treatments of spheroids identified low-dose, strongly synergistic combinations of concentrations from a pair of inhibitors to effectively suppress tumor spheroids growth and block cross-talk between kinase pathways during long-term treatments. Our design-driven approach to determine highly synergistic drug pairs and concentrations against resistance to single-agent treatments offers a valuable tool to prioritize compounds for subsequent animal studies in preclinical tests. This approach will significantly reduce the number of animal studies and accelerate the discovery of effective treatments for clinical use.

\section{ASSOCIATED CONTENT}

\section{S Supporting Information}

The Supporting Information is available free of charge on the ACS Publications website at DOI: 10.1021/acsptsci.9b00042.

Table S1: List and sequences of primers for cell cycle and proliferation genes. Table S2: Design of combination drug treatments with MAPK and PI3K inhibitors (PDF)

\section{AUTHOR INFORMATION}

\section{Corresponding Author}

*Phone: (330) 972-6031. E-mail: tavana@uakron.edu.

ORCID $\odot$

Ramila Joshi: 0000-0001-6351-9051

Hossein Tavana: 0000-0003-3872-1869

\section{Notes}

The authors declare no competing financial interest.

\section{ACKNOWLEDGMENTS}

This work was supported by grants R15CA216413 and R33CA225549 from the National Institute of Health.

\section{REFERENCES}

(1) Yap, T. A., and Workman, P. (2012) Exploiting the Cancer Genome: Strategies for the Discovery and Clinical Development of Targeted Molecular Therapeutics. Annu. Rev. Pharmacol. Toxicol. 52 (1), 549-573. 
(2) Garraway, L. A., and Jänne, P. A. (2012) Circumventing Cancer Drug Resistance in the Era of Personalized Medicine. Cancer Discovery 2 (3), 214-226.

(3) Konieczkowski, D. J., Johannessen, C. M., and Garraway, L. A. (2018) A Convergence-Based Framework for Cancer Drug Resistance. Cancer Cell 33 (5), 801-815.

(4) Dagogo-Jack, I., and Shaw, A. T. (2018) Tumour Heterogeneity and Resistance to Cancer Therapies. Nat. Rev. Clin. Oncol. 15 (2), 81-94.

(5) Sebolt-Leopold, J. S., Dudley, D. T., Herrera, R., Van Becelaere, K., Wiland, A., Gowan, R. C., Tecle, H., Barrett, S. D., Bridges, A., Przybranowski, S., et al. (1999) Blockade of the MAP Kinase Pathway Suppresses Growth of Colon Tumors in Vivo. Nat. Med. 5 (7), 810816.

(6) Yang, H., Higgins, B., Kolinsky, K., Packman, K., Bradley, W. D., Lee, R. J., Schostack, K., Simcox, M. E., Kopetz, S., Heimbrook, D., et al. (2012) Antitumor Activity of BRAF Inhibitor Vemurafenib in Preclinical Models of BRAF-Mutant Colorectal Cancer. Cancer Res. 72 (3), 779-789.

(7) Carson, R., Celtikci, B., Fenning, C., Javadi, A., Crawford, N., Perez-Carbonell, L., Lawler, M., Longley, D. B., Johnston, P. G., and Van Schaeybroeck, S. (2015) HDAC Inhibition Overcomes Acute Resistance to MEK Inhibition in BRAF-Mutant Colorectal Cancer by Downregulation of c-FLIPL. Clin. Cancer Res. 21 (14), 3230-3240.

(8) Corcoran, R. B., Ebi, H., Turke, A. B., Coffee, E. M., Nishino, M., Cogdill, A. P., Brown, R. D., Della Pelle, P., Dias-Santagata, D., Hung, K. E., et al. (2012) EGFR-Mediated Re-Activation of MAPK Signaling Contributes to Insensitivity of BRAF Mutant Colorectal Cancers to RAF Inhibition with Vemurafenib. Cancer Discovery 2 (3), 227-235.

(9) Bon, G., Loria, R., Amoreo, C. A., Verdina, A., Sperduti, I., Mastrofrancesco, A., Soddu, S., Diodoro, M. G., Mottolese, M., Todaro, M., et al. (2017) Dual Targeting of HER3 and MEK May Overcome HER3-Dependent Drug-Resistance of Colon Cancers. Oncotarget 8 (65), 108463-108479.

(10) Sun, C., Hobor, S., Bertotti, A., Zecchin, D., Huang, S., Galimi, F., Cottino, F., Prahallad, A., Grernrum, W., Tzani, A., et al. (2014) Intrinsic Resistance to MEK Inhibition in KRAS Mutant Lung and Colon Cancer through Transcriptional Induction of ERBB3. Cell Rep. 7 (1), 86-93.

(11) Wang, H., Daouti, S., Li, W.-H., Wen, Y., Rizzo, C., Higgins, B., Packman, K., Rosen, N., Boylan, J. F., Heimbrook, D., et al. (2011) Identification of the MEK1(F129L) Activating Mutation as a Potential Mechanism of Acquired Resistance to MEK Inhibition in Human Cancers Carrying the B-RafV600E Mutation. Cancer Res. 71 (16), 5535-5545.

(12) Ahronian, L. G., Sennott, E. M., Van Allen, E. M., Wagle, N., Kwak, E. L., Faris, J. E., Godfrey, J. T., Nishimura, K., Lynch, K. D., Mermel, C. H., et al. (2015) Clinical Acquired Resistance to RAF Inhibitor Combinations in BRAF-Mutant Colorectal Cancer through MAPK Pathway Alterations. Cancer Discovery 5 (4), 358-367.

(13) Corcoran, R. B., Dias-Santagata, D., Bergethon, K., Iafrate, A. J., Settleman, J., and Engelman, J. A. (2010) BRAF Gene Amplification Can Promote Acquired Resistance to MEK Inhibitors in Cancer Cells Harboring the BRAF V600E Mutation. Sci. Signaling 3 (149), ra84ra84.

(14) Comprehensive Molecular Characterization of Human Colon and Rectal Cancer. Nature 2012, 487, 7407, 330-337

(15) Shimizu, T., Tolcher, A. W., Papadopoulos, K. P., Beeram, M., Rasco, D. W., Smith, L. S., Gunn, S., Smetzer, L., Mays, T. A., Kaiser, B., et al. (2012) The Clinical Effect of the Dual-Targeting Strategy Involving PI3K/AKT/MTOR and RAS/MEK/ERK Pathways in Patients with Advanced Cancer. Clin. Cancer Res. 18 (8), 2316-2325.

(16) Flaherty, K. T., Infante, J. R., Daud, A., Gonzalez, R., Kefford, R. F., Sosman, J., Hamid, O., Schuchter, L., Cebon, J., Ibrahim, N., et al. (2012) Combined BRAF and MEK Inhibition in Melanoma with BRAF V600 Mutations. N. Engl. J. Med. 367 (18), 1694-1703.

(17) Molina, A. M., Feldman, D. R., Voss, M. H., Ginsberg, M. S., Baum, M. S., Brocks, D. R., Fischer, P. M., Trinos, M. J., Patil, S., and Motzer, R. J. (2012) Phase 1 Trial of Everolimus plus Sunitinib in
Patients with Metastatic Renal Cell Carcinoma. Cancer 118 (7), $1868-1876$

(18) Ribas, A., Hodi, F. S., Callahan, M., Konto, C., and Wolchok, J. (2013) Hepatotoxicity with Combination of Vemurafenib and Ipilimumab. N. Engl. J. Med. 368 (14), 1365-1366.

(19) Ham, S. L., Joshi, R., Thakuri, P. S., and Tavana, H. (2016) Liquid-Based Three-Dimensional Tumor Models for Cancer Research and Drug Discovery. Exp. Biol. Med. 241 (9), 939-954.

(20) Shahi Thakuri, P., Liu, C., Luker, G. D., and Tavana, H. (2018) Biomaterials-Based Approaches to Tumor Spheroid and Organoid Modeling. Adv. Healthcare Mater. 7 (6), No. 1700980.

(21) Thoma, C. R., Zimmermann, M., Agarkova, I., Kelm, J. M., and Krek, W. (2014) 3D Cell Culture Systems Modeling Tumor Growth Determinants in Cancer Target Discovery. Adv. Drug Delivery Rev. 69-70, 29-41.

(22) Shahi Thakuri, P., Luker, G. D., and Tavana, H. (2019) Cyclical Treatment of Colorectal Tumor Spheroids Induces Resistance to MEK Inhibitors. Transl. Oncol. 12 (3), 404-416.

(23) Ham, S. L., Joshi, R., Luker, G. D., and Tavana, H. (2016) Engineered Breast Cancer Cell Spheroids Reproduce Biologic Properties of Solid Tumors. Adv. Healthcare Mater. 5 (21), 27882798.

(24) Tavana, H., Jovic, A., Mosadegh, B., Lee, Q. Y., Liu, X., Luker, K. E., Luker, G. D., Weiss, S. J., and Takayama, S. (2009) Nanolitre Liquid Patterning in Aqueous Environments for Spatially Defined Reagent Delivery to Mammalian Cells. Nat. Mater. 8 (9), 736-741.

(25) Shahi Thakuri, P., Ham, S. L., Luker, G. D., and Tavana, H. (2016) Multiparametric Analysis of Oncology Drug Screening with Aqueous Two-Phase Tumor Spheroids. Mol. Pharmaceutics 13 (11), 3724-3735.

(26) Shahi Thakuri, P., and Tavana, H. (2017) Single and Combination Drug Screening with Aqueous Biphasic Tumor Spheroids. SLAS Discovery 22 (5), 507-515.

(27) Chou, T.-C. (2010) Drug Combination Studies and Their Synergy Quantification Using the Chou-Talalay Method. Cancer Res. 70 (2), 440-446.

(28) Ham, S. L., Thakuri, P. S., Plaster, M., Li, J., Luker, K. E., Luker, G. D., Tavana, H., et al. (2018) Three-Dimensional Tumor Model Mimics Stromal Breast Cancer Cells Signaling. Oncotarget 9 (1), 249267.

(29) Joshi, R., Buchanan, J. C., and Tavana, H. (2017) SelfRegulatory Factors of Embryonic Stem Cells in Co-Culture with Stromal Cells Enhance Neural Differentiation. Integr. Biol. (Camb). 9 (5), 418-426.

(30) Joshi, R., Thakuri, P. S., Buchanan, J. C., Li, J., and Tavana, H. (2018) Microprinted Stem Cell Niches Reveal Compounding Effect of Colony Size on Stromal Cells-Mediated Neural Differentiation. Adv. Healthcare Mater. 7 (5), 1700832.

(31) Atefi, E., Joshi, R., Mann, J. A., and Tavana, H. (2015) Interfacial Tension Effect on Cell Partition in Aqueous Two-Phase Systems. ACS Appl. Mater. Interfaces 7 (38), 21305-21314.

(32) Lemmo, S., Atefi, E., Luker, G. D., and Tavana, H. (2014) Optimization of Aqueous Biphasic Tumor Spheroid Microtechnology for Anti-Cancer Drug Testing in 3D Culture. Cell. Mol. Bioeng. 7 (3), 344-354.

(33) Atefi, E., Lemmo, S., Fyffe, D., Luker, G. D., and Tavana, H. (2014) High Throughput, Polymeric Aqueous Two-Phase Printing of Tumor Spheroids. Adv. Funct. Mater. 24 (41), 6509-6515.

(34) Thakuri, P. S., Gupta, M., Plaster, M., and Tavana, H. (2019) Quantitative Size-Based Analysis of Tumor Spheroids and Responses to Therapeutics. Assay Drug Dev. Technol. 17 (3), 140-149.

(35) Ham, S. L., Atefi, E., Fyffe, D., and Tavana, H. (2015) Robotic Production of Cancer Cell Spheroids with an Aqueous Two-Phase System for Drug Testing. J. Visualized Exp. 98, No. e52754.

(36) Ahmed, D., Eide, P. W., Eilertsen, I. A., Danielsen, S. A., Eknæs, M., Hektoen, M., Lind, G. E., and Lothe, R. A. (2013) Epigenetic and Genetic Features of 24 Colon Cancer Cell Lines. Oncogenesis 2, No. e71. 
(37) Hoeflich, K. P., Merchant, M., Orr, C., Chan, J., Den Otter, D., Berry, L., Kasman, I., Koeppen, H., Rice, K., Yang, N.-Y., et al. (2012) Intermittent Administration of MEK Inhibitor GDC-0973 plus PI3K Inhibitor GDC-0941 Triggers Robust Apoptosis and Tumor Growth Inhibition. Cancer Res. 72 (1), 210-219.

(38) Sos, M. L., Fischer, S., Ullrich, R., Peifer, M., Heuckmann, J. M., Koker, M., Heynck, S., Stuckrath, I., Weiss, J., Fischer, F., et al. (2009) Identifying Genotype-Dependent Efficacy of Single and Combined PI3K- and MAPK-Pathway Inhibition in Cancer. Proc. Natl. Acad. Sci. U. S. A. 106 (43), 18351-18356.

(39) Balmanno, K., Chell, S. D., Gillings, A. S., Hayat, S., and Cook, S. J. (2009) Intrinsic Resistance to the MEK1/2 Inhibitor AZD6244 (ARRY-142886) Is Associated with Weak ERK1/2 Signalling and/or Strong PI3K Signalling in Colorectal Cancer Cell Lines. Int. J. Cancer 125 (10), 2332-2341.

(40) Mirzoeva, O. K., Das, D., Heiser, L. M., Bhattacharya, S., Siwak, D., Gendelman, R., Bayani, N., Wang, N. J., Neve, R. M., Guan, Y., et al. (2009) Basal Subtype and MAPK/ERK Kinase (MEK)Phosphoinositide 3-Kinase Feedback Signaling Determine Susceptibility of Breast Cancer Cells to MEK Inhibition. Cancer Res. 69 (2), $565-572$.

(41) Zhou, B. P., Liao, Y., Xia, W., Spohn, B., Lee, M.-H., and Hung, M.-C. (2001) Cytoplasmic Localization of P21Cip1/WAF1 by AktInduced Phosphorylation in HER-2/Neu-Overexpressing Cells. Nat. Cell Biol. 3 (3), 245-252.

(42) Rössig, L., Badorff, C., Holzmann, Y., Zeiher, A. M., and Dimmeler, S. (2002) Glycogen Synthase Kinase-3 Couples AKTDependent Signaling to the Regulation of P21 ${ }^{\text {Cip } 1}$ Degradation. J. Biol. Chem. 277 (12), 9684-9689.

(43) Rossig, L., Jadidi, A. S., Urbich, C., Badorff, C., Zeiher, A. M., and Dimmeler, S. (2001) Akt-Dependent Phosphorylation of P21Cip1 Regulates PCNA Binding and Proliferation of Endothelial Cells. Mol. Cell. Biol. 21 (16), 5644-5657.

(44) Maira, S.-M., Stauffer, F., Schnell, C., and García-Echeverría, C. (2009) PI3K Inhibitors for Cancer Treatment: Where Do We Stand? Biochem. Soc. Trans. 37 (1), 265-272.

(45) Haagensen, E. J., Kyle, S., Beale, G. S., Maxwell, R. J., and Newell, D. R. (2012) The Synergistic Interaction of MEK and PI3K Inhibitors Is Modulated by MTOR Inhibition. Br. J. Cancer 106 (8), 1386-1394.

(46) Kong, D., Yaguchi, S., and Yamori, T. (2009) Effect of ZSTK474, a Novel Phosphatidylinositol 3-Kinase Inhibitor, on DNADependent Protein Kinase. Biol. Pharm. Bull. 32 (2), 297-300.

(47) Engelman, J. A., Chen, L., Tan, X., Crosby, K., Guimaraes, A. R., Upadhyay, R., Maira, M., McNamara, K., Perera, S. A., Song, Y., et al. (2008) Effective Use of PI3K and MEK Inhibitors to Treat Mutant Kras G12D and PIK3CA H1047R Murine Lung Cancers. Nat. Med. 14 (12), 1351-1356.

(48) Gong, S., Xu, D., Zhu, J., Zou, F., and Peng, R. (2018) Efficacy of the MEK Inhibitor Cobimetinib and Its Potential Application to Colorectal Cancer Cells. Cell. Physiol. Biochem. 47 (2), 680-693.

(49) Chang, F., Lee, J. T., Navolanic, P. M., Steelman, L. S., Shelton, J. G., Blalock, W. L., Franklin, R. A., and McCubrey, J. A. (2003) Involvement of PI3K/Akt Pathway in Cell Cycle Progression, Apoptosis, and Neoplastic Transformation: A Target for Cancer Chemotherapy. Leukemia 17 (3), 590-603.

(50) Haagensen, E. J., Thomas, H. D., Wilson, I., Harnor, S. J., Payne, S. L., Rennison, T., Smith, K. M., Maxwell, R. J., and Newell, D. R. (2013) The Enhanced in Vivo Activity of the Combination of a MEK and a PI3K Inhibitor Correlates with [18F]-FLT PET in Human Colorectal Cancer Xenograft Tumour-Bearing Mice. PLoS One 8 (12), No. e81763.

(51) Shimizu, T., Tolcher, A. W., Papadopoulos, K. P., Beeram, M., Rasco, D. W., Smith, L. S., Gunn, S., Smetzer, L., Mays, T. A., Kaiser, B., et al. (2012) The Clinical Effect of the Dual-Targeting Strategy Involving PI3K/AKT/MTOR and RAS/MEK/ERK Pathways in Patients with Advanced Cancer. Clin. Cancer Res. 18 (8), 2316-2325. (52) Raja, M., Zverev, M., Seipel, K., Williams, G. T., Clarke, A. R., and Shaw, P. H. S. (2015) Assessment of the in Vivo Activity of PI3K and MEK Inhibitors in Genetically Defined Models of Colorectal Cancer. Mol. Cancer Ther. 14 (10), 2175-2186. 